\title{
A Common Cold Virus, Rhinovirus 16, Potentiates Airway Inflammation after Segmental Antigen Bronchoprovocation in Allergic Subjects
}

\author{
William J. Calhoun, ${ }^{\star}$ Elliot C. Dick, ${ }^{\ddagger}$ Lawrence B. Schwartz, ${ }^{\S}$ and William W. Busse" \\ "Department of Medicine, Section of Allergy \& Clinical Immunology and ${ }^{\ddagger}$ Department of Preventive Medicine, University of Wisconsin, \\ Madison, Wisconsin 53706; *Division of Pulmonary, Allergy, and Critical Care Medicine, University of Pittsburgh, Pittsburgh, \\ Pennsylvania 15261; and the ${ }^{\S}$ Division of Rheumatology, Allergy \& Immunology, Medical College of Virginia, Richmond, Virginia 23298
}

\begin{abstract}
Many patients with asthma have increased wheezing with colds. We hypothesized that rhinovirus colds might increase asthma by augmenting airway allergic responses (histamine release and eosinophil influx) after antigen challenge. Seven allergic rhinitis patients and five normal volunteers were infected with rhinovirus type 16 (RV16) and evaluated by segmental bronchoprovocation and bronchoalveolar lavage. Segmental challenge with saline and antigen was performed 1 mo before infection, during the acute infection, and 1 mo after infection. Lavage was performed immediately and 48 $h$ after antigen challenge. Data were analyzed by two-way analysis of variance, and a $P$ value of $\leq 0.05$ was considered to be significant. All volunteers inoculated with RV16 developed an acute respiratory infection. BAL fluid obtained from allergic rhinitis subjects during the acute viral infection, and 1 mo after infection, showed the following significant RV16-associated changes after antigen challenge: (a) an enhanced release of histamine immediately after local antigen challenge; $(b)$ persistent histamine leak $48 \mathrm{~h}$ afterwards; and (c) a greater recruitment of eosinophils to the airway $48 \mathrm{~h}$ after challenge. These changes were not seen in non-allergic volunteers infected with RV16 and challenged with antigen, nor in allergic volunteers repetitively challenged with antigen but not infected with RV16, nor in RV16 infected allergic volunteers sham challenged with saline. We conclude that rhinovirus upper respiratory infection significantly augments immediate and late allergic responses in the airways of allergic individuals after local antigen challenge. These data suggest that one mechanism of increased asthma during a cold is an accentuation of allergic responses in the airway which may then contribute to bronchial inflammation. (J. Clin. Invest. 1994. 94:2200-2208.) Key words: bronchoalveolar lavage $\cdot$ asthma $\cdot$ eosinophils - histamine • inflammation • cytokines $\cdot$ common cold
\end{abstract}

\section{Introduction}

Rhinoviruses cause common colds which often provoke wheezing in patients with asthma (1-3). The wheezing can be severe,

Address correspondence to William J. Calhoun, M.D., Associate Professor of Medicine, Division of Pulmonary, Allergy, and Critical Care Medicine, 440 Scaife Hall, 3550 Terrace St., Pittsburgh, PA 15261.

Received for publication 22 February 1994 and in revised form 21 July 1994.

J. Clin. Invest.

(c) The American Society for Clinical Investigation, Inc.

$0021-9738 / 94 / 12 / 2200 / 09 \quad \$ 2.00$

Volume 94, December 1994, 2200-2208 and may persist considerably longer than the clinical signs and symptoms of fever, nasal congestion, and rhinorrhea $(2,3)$. Recent observations have confirmed that rhinoviruses are the most common infectious exacerbants in adult patients, and that these agents are commonly associated with significant reductions in peak expiratory flow rates (4). In addition, Duff and colleagues (5) have convincingly shown that both atopy and viral infections are risk factors for wheezing in older $(>2$ yr) children, and that rhinoviral infections provoke wheezing predominantly in allergic patients. The relative segregation of viral-induced wheezing to allergic subjects implies that important interactions may exist between viral infections and allergic reactions. However, the precise mechanisms by which rhinoviral upper respiratory illnesses exacerbate asthma have yet to be fully established. We have developed an in vivo human model which allows assessment of airway physiology and biology during viral upper respiratory tract infections. In previous studies, allergic rhinitis patients were intranasally inoculated with live rhinovirus type 16 (RV16). ${ }^{1}$ During the acute infection, and for weeks afterwards, these allergic individuals showed increased airway responsiveness to inhaled histamine, methacholine, and antigen, and an increased likelihood for late allergic (inflammatory) responses after inhaled antigen challenge (6). Subsequently, we showed that RV16 infection was associated with higher concentrations of plasma histamine after antigen challenge during RV16 infection in subjects with new late asthmatic responses (7). These observations suggest an important relationship between viral infections and the potentiation of allergic responses, including inflammation, in the airway.

To study the mechanisms of allergic airway responses in more detail, we $(8,9)$ and others $(10,11)$ have used fiberoptic bronchoscopy to perform segmental bronchoprovocation with antigen and bronchoalveolar lavage (BAL). For segmental challenges, a bronchoscope is introduced into the airways, bronchial segments identified, and an allergen administered directly onto the airway mucosa. With this technique, the immediate response is characterized by a brisk rise in the mast cell mediators histamine and tryptase. In contrast, BAL fluid obtained $48 \mathrm{~h}$ after antigen challenge in allergic, but not normal, individuals is highly cellular, eosinophil-rich, and contains a variety of cytokines $(9,12)$. Based upon our earlier observations that a RV16 infection increases bronchial responsiveness, plasma histamine levels, and late asthmatic responses to airway antigen challenges, we have used segmental antigen challenge and BAL to test our hypothesis that an RV upper viral respiratory illness enhances the airway allergic inflammatory response to antigen,

1. Abbreviations used in this paper: ANOVA, analysis of variance; BAL, bronchoalveolar Lavage; RV16, Rhinovirus, type 16. 
Table I. Virologic and Clinical Results of Inoculation with RV16*

\begin{tabular}{lllccc}
\hline \multicolumn{1}{c}{ Subject } & Serum titers & $\begin{array}{c}\text { Nasal } \\
\text { virus }\end{array}$ & BAL virus & $\begin{array}{c}\text { Cell pellet } \\
\text { virus }\end{array}$ & Max Sx score \\
\hline 1 & $1.4 \rightarrow 45$ & 4 & ND & ND & 6 \\
2 & $<1 \rightarrow 5.6$ & 5 & 1 & ND & 10 \\
3 & $1.4 \rightarrow 32$ & 4.5 & ND & ND & 5 \\
4 & $<1 \rightarrow 2.8$ & 4 & ND & ND & 15 \\
5 & $<1 \rightarrow 2.8$ & 3.5 & ND & ND & 9 \\
Median & $<1 \rightarrow 5.6$ & 4.5 & ND & ND & 9 \\
A & $<1 \rightarrow 5.6$ & 5 & ND & ND & 10 \\
B & $<1 \rightarrow 22.7$ & 4 & ND & ND & 13 \\
D & $<1 \rightarrow 11$ & 5.5 & ND & ND & 8 \\
E & $1 \rightarrow 4$ & 5 & ND & ND & 4 \\
F & $<1 \rightarrow 8$ & 2.5 & ND & ND & 4 \\
G & $<1 \rightarrow 32$ & 4 & ND & ND & 10 \\
H & $<1 \rightarrow 45$ & 3.5 & ND & ND & 15 \\
Median & $<1 \rightarrow 9.5$ & 4 & ND & ND & 9.5 \\
Difference & NS & NS & NS & NS & NS \\
\hline
\end{tabular}

* Subjects 1-5, normal volunteers; subjects A-H, allergic rhinitis. None of these parameters differed significantly between normal and allergic subjects. Serum titer: antibody against RV16 in pre-infection and postinfection study periods (all sera were devoid $[<1: 2]$ of RV16 antibody during initial screening). Baseline serology demonstrated small amounts of RV16 antibody in subjects 1, 3, and E; all three had mild colds as judged by their symptoms. Nasal virus: highest log TCID50 RV16 in nasal wash during acute infection study period. BAL virus: RV16 in BAL fluid supernatant; one plaque forming unit was detected in $0.2 \mathrm{ml}$ of undiluted BAL fluid from subject 2. Cell pellet virus: RV16 in BAL cells. Max Sx score: maximum symptom score during cold. See text. $N D$, no virus detected.

including histamine release and eosinophil influx into the airway.

\section{Methods}

Subjects. Seven allergic rhinitis subjects and five normal volunteers were selected for study and RV16 inoculation. An eighth allergic subject was recruited, but excluded from analysis because a contaminating rhinovirus was detected in nasal washes during the Pre-infection period. These subjects had no neutralizing antibody to RV16 in undiluted serum when screened before this study (Table I). Allergic rhinitis subjects were characterized by seasonal or episodic rhinitis (nasal congestion, rhinorrhea, or excessive sneezing) (13) and by a positive skin test (prickpuncture method; 1:20 wt/vol) to one or more common inhalant allergens, including cat (one individual), grass (one individual), or ragweed (five individuals) antigens (Greer Laboratories, Lenoir, NC). All were studied during periods when they were not symptomatic with rhinitis. The five normal volunteers were free of cardiopulmonary complaints or nasal symptoms, and had negative skin tests to the same battery of aeroallergens. These individuals were selected to control for the effect of RV16 infection on segmental antigen challenge with ragweed antigen in non-allergic individuals. An additional group of four allergic subjects was also studied to provide additional controls; they were not inoculated with RV16 but underwent repetitive seg-

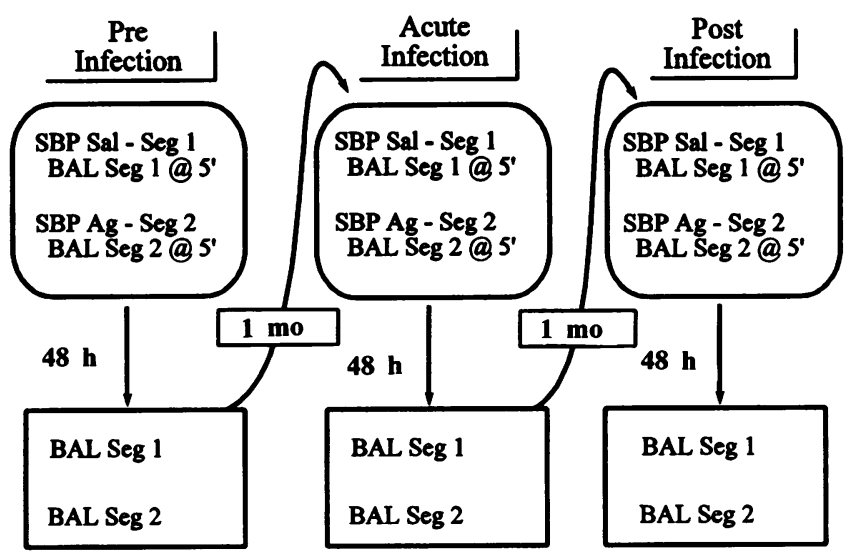

Figure 1. Experimental design. Five normal and seven allergic rhinitis subjects were studied. The pulmonary response to antigen challenge was examined during three study periods: 1 mo before (Pre Infection), during (Acute Infection), and 1 mo after (Post Infection) experimental infection with rhinovirus type 16. On the first of two study days in each period, a bronchoscopy was performed, and segmental challenge with saline was accomplished, followed by bronchoalveolar lavage $5 \mathrm{~min}$ later. Segmental challenge with antigen and subsequent BAL were then performed similarly in a separate bronchopulmonary segment. $48 \mathrm{~h}$ later, the two segments were lavaged again.

mental antigen challenge and lavage. These individuals served as controls to determine the effect of repetitive antigen challenge on the airway response to allergen. All participants were nonsmokers, and each gave consent to a protocol approved by the University of Wisconsin Human Subjects Committee.

Experimental design. Each subject underwent screening spirometry, skin testing, and aerosol antigen challenge on entry to the study. At least $1 \mathrm{mo}$ was then allowed to elapse before initiating the bronchoscopic studies; the experimental design is schematized in Fig. 1. Each subject inoculated with RV16 was studied by bronchoscopy, segmental antigen challenge, immediate BAL, and late $(48 \mathrm{~h}) \mathrm{BAL}$ during each of three study periods: (1) pre-infection, (2) acute infection, and (3) post-infection. These three study periods were separated by $1 \mathrm{mo}$. At bronchoscopy, two airway segments were identified, one in the right lung and one in left lung. The first segment was challenged with saline and the second segment was challenged with antigen. During each study period, segmental challenge with saline and antigen, and immediate BAL were performed ("immediate response"), followed $48 \mathrm{~h}$ later by BAL in each of the two segments ("late response"). An identical approach was followed in the normal volunteers. In four allergic subjects not inoculated with RV16, segmental challenge with antigen, and immediate and late BALs were performed on two separate occasions, each separated by at least $28 \mathrm{~d}$.

RV16 inoculation, confirmation of infection, and assessment of cold severity. The technique for RV16 inoculation used previously described methods $(7,14)$. Viral respiratory tract infections were induced by inoculations on two consecutive days. Inoculations entailed instilling $0.25 \mathrm{ml}$ of RV16 suspension into each nostril by pipette (500-18,000 tissue culture infective doses $\left[\mathrm{TCID}_{50}\right]$ ), and spraying approximately the same amount into each nostril with a compressor-driven atomizer (No. 286; DeVilbiss Co., Somerset, PA) which produces a coarse mist of droplets which largely deposit on the nasal mucosa. Previous 
studies have demonstrated that this dual-inoculation strategy maximizes the efficiency of infection and resultant common cold symptoms $(6,7,14)$. RV16 inoculations were completed $48 \mathrm{~h}$ before the first bronchoscopy and segmental antigen challenge. Cold symptoms were evaluated for $7 \mathrm{~d}$ with a questionnaire that was completed by the participant hourly during waking hours as previously described (14). Eight signs and symptoms were evaluated on a four-point scale $(0-3)$ that included cough, nasal discharge, sneezing, nasal congestion, headache, malaise, chills, and fever which occurred at any time during that 24 -h period. Symptom scores of $<7$ defined a mild cold, 7-11 a moderate cold, and 12 or greater a severe cold (14). Maximum symptom score was the highest daily score recorded at any time during the cold. It was not possible to distinguish bronchoscopy-related cough, nasal congestion, and fever from that related to viral infection. Nasal washing, virus titration, and serum titer determinations were performed as previously described $(7,14)$. Unfractionated BAL fluid, mechanically disrupted BAL cells, and BAL fluid supernatants were inoculated onto human diploid (WI-38) cell lines and evaluated for RV16 infection by criteria previously described (14).

Determination of ragweed antigen dose for segmental challenge. The physiologic response of the airway to antigen was determined during the screening period at least 1 mo before the pre-infection study period by standard bronchial provocation techniques, as previously described $(15,16)$. Briefly, increasing concentrations of antigen were delivered by a nebulizing dosimeter (Rosenthal-French, Laboratory for Applied Immunology, Baltimore, MD) until (a) the $\mathrm{FEV}_{1}$ had fallen at least $20 \%$ from the baseline measurement, or $(b)$ a maximum of five breaths of 10,000 protein nitrogen $\mathrm{U} / \mathrm{ml}$ (1833 cumulative units) had been given. The dose of antigen required to produce a $20 \%$ decrease in $\mathrm{FEV}_{1}$ was calculated by linear regression $\left(\mathrm{FEV}_{1}\right.$ vs the $\log$ of cumulative dose of antigen) of the log-normal curve using a commercial analysis package for microcomputers (PD20; Madison Scientific Software, Wexford, PA) and defined as the provocative dose of antigen $\left[\mathrm{PD}_{20}\right]$.

Bronchoscopy, bronchoalveolar lavage, and segmental bronchoprovocation. Spirometry was obtained before premedications and local anesthesia. Bronchoalveolar lavage was performed as previously described $(8,15,17)$. After premedication with atropine ( $0.6 \mathrm{mg} \mathrm{IM}$ ) and midazolam (1.25 mg IM), nasopharyngeal anesthesia was achieved with topical cocaine $(4 \%)$ and lidocaine $(1 \%)$. Supplemental oxygen (3 liters/min) was provided, and pulse oximetry was monitored throughout. The bronchoscope was introduced into the airway and wedged into a segmental or subsegmental bronchus. Saline challenge was performed by instilling into a bronchopulmonary segment, or subsegment, $10 \mathrm{ml}$ of saline $(0.9 \% \mathrm{NaCl})$. Subsequently, the bronchoscope channel was flushed with $5 \mathrm{ml}$ of air while maintaining the wedged position. After a dwell time of $5 \mathrm{~min}, \mathrm{BAL}$ was performed by instilling and immediately withdrawing two 60 -ml aliquots of warm $\left(37^{\circ} \mathrm{C}\right)$ sterile saline (immediate response). Segmental antigen challenge was performed by instilling into a separate, contralateral bronchopulmonary segment or subsegment, a quantity of antigen equal to $20 \%$ of the previously determined provocative dose of antigen $\left(\mathrm{PD}_{20}\right)$. This antigen dose was diluted in $10 \mathrm{ml}$ sterile saline. For example, if a cumulative aerosol dose of 150 protein nitrogen units (PNU) of ragweed antigen produced a $20 \%$ fall in $\mathrm{FEV}_{1}$, the dose of antigen for segmental challenge would be 30 PNU (20\% of
$150)$ in $10 \mathrm{ml}$. After instillation, the channel was flushed with $5 \mathrm{ml}$ of air. After a 5-min dwell time, BAL was performed for immediate-response measurements. Saline challenge and immediate BAL always preceded antigen challenge and BAL to avoid any possible antigen carry-over. Within $1 \mathrm{~h}$ of completing the bronchoscopy, spirometry was repeated. $48 \mathrm{~h}$ later, repeat spirometry, bronchoscopy, and BAL to determine the late response were performed in the same manner. Care was exercised to identify and enter precisely the same anatomic segments used for study on the first day.

$B A L$ processing. The two BAL fluid aliquots from each individual segment were pooled for processing, and the volume measured. Cells were recovered by centrifugation, washed once in PBS, and counted using a hemocytometer. The supernatant fluid was then frozen $\left(-70^{\circ} \mathrm{C}\right)$ until analyzed for histamine, tryptase, protein, and cytokines. Cytofuge preparations were airdried, methanol fixed, and stained with Diff-Quick (Scientific Products, Chicago, IL). At least 300 leukocytes were identified on each smear as alveolar macrophages, lymphocytes, eosinophils, or neutrophils. Smears were stained with nonspecific esterase to aid in differentiating small macrophages from lymphocytes as required. Histamine was determined using a radioenzymatic $N$-methyltransferase assay $(9,18)$. The limit of detectability for histamine was $30 \mathrm{pg} / \mathrm{ml}$. Tryptase was measured with a limit of detectability of $0.1 \mathrm{ng} / \mathrm{ml}$ by a sandwich ELISA in samples concentrated up to 20 -fold by ultrafiltration using a Centricon-10 chamber (Amicon Corp., Danvers, MA) (19). TNF- $\alpha$ levels were determined in unconcentrated BAL fluids using a four-layer sandwich ELISA $(20,21)$. Standard curves for this assay were prepared using recombinant cytokine. The sensitivity of the TNF- $\alpha$ assay was $0.2 \mathrm{ng} / \mathrm{ml}$, and dayto-day variability (coefficient of variation) approximated $10 \%$. Total protein was measured with a detection limit of $20 \mu \mathrm{g} / \mathrm{ml}$ by a microtiter modification of the Lowry assay $(8,15)$. All data were expressed and analyzed statistically as absolute concentrations, as currently recommended (17).

Statistical analysis. Data were analyzed using a statistical package for microcomputers (SigmaStat 1.01; Jandel Scientific, San Rafael, CA) for the effects of RV16 infection and allergic state by two-way analysis of variance (ANOVA) for repeated measures using a general linear method (22). Because the data distributions failed the test for normality, logarithmic transformations were used in the ANOVAs (23). Unless otherwise specified, all comparisons reported are the result of this ANOVA. We report (a) the effect of RV16 infection, $(b)$ the effect of atopic status, and $(c)$ the difference in response to RV16 of atopics and allergics. When a significant ANOVA was identified, post hoc testing was conducted by the Student-NewmanKeuls test. Summary data are presented as the mean \pm the standard error of the mean. Undetectable values were analyzed statistically by substituting the limit of detectability of the particular assay. A $P$ value of $\leq 0.05$ was considered to be significant (23).

\section{Results}

We report the results of this investigation in four sections, which are briefly summarized here. (a) All 12 subjects inoculated became infected and symptomatic with colds. No differences in frequency or severity of colds were apparent between normal and allergic subjects. (b) RV16 infection did not significantly 
Table II. Effects of RVI6 Inoculation on Airway Physiology*

\begin{tabular}{lcrrrr}
\hline Parameter & Group & \multicolumn{1}{c}{ Baseline } & Inoculation & Recovery & $P^{\ddagger}$ \\
\hline FEV $_{1}$ & NV & $105.6 \pm 6.9$ & $104.3 \pm 6.2$ & $104.3 \pm 4.8$ & 0.77 \\
& AR & $109.4 \pm 5.1$ & $105.8 \pm 4.6$ & $105.6 \pm 4.6$ & 0.15 \\
FVC & NV & $97.4 \pm 4.4$ & $96.5 \pm 4.4$ & $100.0 \pm 4.8$ & 0.19 \\
& AR & $108.7 \pm 3.9$ & $104.3 \pm 2.9$ & $104.9 \pm 3.2$ & 0.14
\end{tabular}

* FEV, FVC, before bronchoscopy, percent predicted, mean \pm SEM.

‡ Statistical significance for effect of RV16 inoculation, ANOVA. NV, normal volunteer; $A R$, allergic rhinitis.

change airway physiology or BAL volume recoveries. (c) RV16 infection significantly increased BAL histamine concentrations 5 min after segmental antigen challenge in allergic but not normal subjects; BAL tryptase concentrations at the same time increased, but were not significantly different. Total and differential cell counts and TNF- $\alpha$ concentrations determined $5 \mathrm{~min}$ after antigen challenge were not affected by RV16 infection in either allergic or normal subjects. (d) $48 \mathrm{~h}$ after segmental antigen challenge of allergic but not normal subjects, RV16 infection was associated with significantly increased BAL concentrations of histamine, recovery of eosinophils, and levels of TNF- $\alpha$.

\section{Confirmation of rhinovirus 16 infection}

All seven allergic and five normal subjects inoculated with RV16 became infected as defined by a fourfold or greater rise in RV16 titer (pre-infection to post-infection), or recovery of at least $10^{2.5}$ TCID $_{50}$ viral particles per milliliter of nasal wash (Table I). Colds were also evident clinically as all subjects reported rhinorrhea and nasal congestion even before bronchoscopy and segmental antigen challenge. Of the allergic subjects, two had mild colds, three had moderate colds, and two had severe colds. The normal volunteers inoculated with RV16 also developed colds and had similar laboratory responses and clinical symptoms. There were no significant differences in the severity of symptoms and signs, serum antibody titers, or nasal virus shedding between normal and allergic groups. Thus, allergic and normal subjects developed comparable RV16 upper respiratory tract infections. The atomizer used to inoculate subjects produced a coarse spray rather than an inspirable mist. A quantitative analysis of the particle size distribution was not available because the device was not designed to produce inspirable particles. Thus, it is not possible to exclude the possibility that some RV16 inoculum was inspired. However, in only one subject (normal volunteer No. 2) was RV16 detected by culture from BAL materials, and that was at very low titer (Table I). Thus, culture positive viral recovery after inoculation was prominently identified from the nose, but was not observed from the lower respiratory tract.

\section{Effect of RV16 infection on airway physiology and BAL} volume recovery

Physiology. Infection with RV16 did not significantly change airway physiology measured immediately before the first BAL and segmental antigen challenge in either normal or allergic subjects (Table II). Moreover, in both allergic and normal subjects, the $\mathrm{FEV}_{1}$ and FVC before bronchoscopy was similar on
Table III. BAL Parameters Immediately after Antigen Challenge*

\begin{tabular}{ccccc}
\hline Parameter & Group & Pre-infection $^{\ddagger}$ & Acute infection & Post-infection \\
\hline $\begin{array}{c}\text { Total cells } \\
\text { (millions) }\end{array}$ & $\mathrm{NV}^{+}$ & $15.6 \pm 1.9$ & $18.6 \pm 3.9$ & $16.8 \pm 2.8$ \\
& $\mathrm{AR}$ & $19.6 \pm 5.1$ & $21.0 \pm 3.4$ & $25.3 \pm 4.4$ \\
EOS & $\mathrm{NV}$ & $0.009 \pm 0.009$ & $0.051 \pm 0.033$ & $0.24 \pm 0.14$ \\
$\quad($ millions) & & & & \\
& $\mathrm{AR}$ & $0.046 \pm 0.027$ & $0.44 \pm 0.27$ & $0.23 \pm 0.095$ \\
NEUT & $\mathrm{NV}$ & $0.055 \pm 0.029$ & $2.09 \pm 2.05$ & $0.165 \pm 0.110$ \\
$\quad($ millions) & & & & \\
& $\mathrm{AR}$ & $0.083 \pm 0.035$ & $0.24 \pm 0.12$ & $0.42 \pm 0.20$ \\
LYMPHS & $\mathrm{NV}$ & $0.38 \pm 0.16$ & $0.16 \pm 0.13$ & $0.55 \pm 0.22$ \\
$\quad($ millions $)$ & & & & \\
& $\mathrm{AR}$ & $1.22 \pm 0.78$ & $0.41 \pm 0.12$ & $1.59 \pm 0.17$ \\
AM & $\mathrm{NV}$ & $15.1 \pm 1.8$ & $16.3 \pm 2.8$ & $15.7 \pm 2.4$ \\
$\quad($ millions $)$ & & & & \\
& $\mathrm{AR}$ & $18.2 \pm 5.0$ & $19.8 \pm 3.5$ & $23.0 \pm 7.0$ \\
Total protein & $\mathrm{NV}$ & $85.4 \pm 17.7$ & $73.9 \pm 9.9$ & $66.4 \pm 17.2$ \\
$\quad(\mu \mathrm{g} / \mathrm{ml})$ & $\mathrm{AR}$ & $103.0 \pm 22.0$ & $98.6 \pm 23.8$ & $92.0 \pm 14.6$ \\
\hline
\end{tabular}

* Data expressed as mean \pm SEM. $\quad{ }^{\ddagger}$ Neither the effect of RV Infection nor the effect of atopic status were significant for any of the parameters listed by two-way ANOVA with repeated measures. $N V$, normal volunteer; $A R$, allergic rhinitis; EOS, eosinophils; $N E U T$, neutrophils;

$L Y M P H S$, lymphocytes; $A M$, alveolar macrophages.

both days ( $P>0.7$, ANOVA, all comparisons; data not shown). All volunteers tolerated the segmental antigen challenges without difficulty. There were no acute bronchial responses to the segmental antigen challenge which necessitated either interruption or cessation of bronchoscopy at any of the study periods.

$B A L$ volume. Lavage volume recovery was similar at all time points of study in both the allergic and normal subjects (data not shown). Consequently, the measured concentrations are reported without notation to, or correction for, the $\mathrm{BAL}$ volume recovery as currently recommended (17).

\section{Effect of RV16 infection on immediate responses}

There were no significant effects of RV16 infection or allergic status on total protein concentrations, total cell recovery, recovery of alveolar macrophages, lymphocytes, neutrophils, and eosinophils (Table III), or the concentration of TNF- $\alpha$ in immediate response $\mathrm{BAL}$ fluids obtained $5 \mathrm{~min}$ after antigen challenge ( $P>0.08$, ANOVA, all comparisons). However, TNF- $\alpha$ was detectable more frequently $5 \mathrm{~min}$ after antigen challenge in allergics (17/21 samples) than normals (9/15 samples), and the concentrations of this cytokine were significantly greater in allergics $(6.6 \pm 2.1$ vs. $1.7 \pm 2.5 \mathrm{ng} / \mathrm{ml} ; P<0.05)$. No release of histamine or tryptase was induced by saline instillation in either normal volunteers or allergic rhinitis patients (data not shown).

Histamine. Histamine was detectable in BAL fluid from $7 /$ 15 normal samples, and in 19/21 fluids from allergic subjects. Histamine concentrations after antigen challenge in the immediate response were significantly augmented by RV16 infection in allergic, but not normal subjects $(P<0.04$, ANOVA; allergic subjects: pre-infection $1,148 \pm 839$, acute infection $4,522 \pm 3,284$, post-infection $5,990 \pm 3,757 \mathrm{pg} / \mathrm{ml} ; P<0.04$, ANOVA; normal subjects: pre-infection $48 \pm 15$, acute infection 
$61 \pm 37$, post-infection $54 \pm 33 \mathrm{pg} / \mathrm{ml}$, respectively; $P>0.30$, ANOVA). Moreover, in allergic subjects, the concentrations of histamine during the acute infection and post-infection periods were significantly higher than those in the pre-infection period ( $P<0.05$, Newman-Keuls, both comparisons). As expected, there was a significant effect of atopy, as histamine concentrations after antigen challenge in immediate response BAL fluids were higher in allergics $(3,887 \pm 1,663 \mathrm{pg} / \mathrm{ml})$ than in normal subjects $(55 \pm 8 \mathrm{pg} / \mathrm{ml} ; P<0.001$, ANOVA); however, the difference in response to RV16 infection between atopic and nonatopic subjects did not reach statistical significance $(P=0.14$, ANOVA).

In four allergic subjects who underwent repetitive segmental challenges with antigen, the BAL fluid histamine levels in the immediate response were similar at both challenges (first challenge: $2,132 \pm 1,136 \mathrm{pg} / \mathrm{ml}$ vs. second challenge: $1,045 \pm 607 \mathrm{pg} /$ $\mathrm{ml}, P=\mathrm{NS}$ ).

Tryptase. Qualitatively similar patterns were noted for BAL tryptase concentrations immediately after antigen challenge. Tryptase was undetectable in $5 / 15$ normal samples, and $\leq 0.3$ $\mathrm{ng} / \mathrm{ml}$ in the remainder. In contrast, tryptase could be detected in 18/21 samples from allergic subjects. Little tryptase was identified in immediate response BAL fluids from normal volunteers; significantly higher concentrations were measured in allergic subjects (allergic: $2.1 \pm 0.6$ vs. Normal: $0.2 \pm 0.03 \mathrm{ng} / \mathrm{ml}$, $P<0.03$ ANOVA). Tryptase concentrations in immediate response BAL fluids from normal subjects were comparable in each study period and were not significantly influenced by RV16 infection (change with RV16 infection $\leq 0.2 \mathrm{ng} / \mathrm{ml}$ ). Infection with RV16 was associated with a progressive rise in immediate response tryptase concentrations in allergic subjects (pre-infection: $1.3 \pm 0.6$, acute infection: $1.8 \pm 0.7$, post-infection: $3.0 \pm 1.6)$. This rise, however, did not reach statistical significance $(P>0.1$, ANOVA).

Repetitive antigen challenges in four allergic subjects did not alter the immediate tryptase response after segmental allergen challenge in subjects not infected with RV16 (first challenge: $0.6 \pm 0.2$ vs. second challenge: $0.5 \pm 0.1 \mathrm{ng} / \mathrm{ml}, P=\mathrm{NS}$ ).

Summary of effects of RVI6 Infection on immediate responses. Histamine release into the airway immediately after local antigen challenge was significantly potentiated by RV16 infection in allergic, but not normal subjects during and after RV16 infection compared with pre-infection values. No changes related to RV16 infection were observed in total cell recovery, TNF- $\alpha$ levels, tryptase concentrations, or airway physiology.

\section{Effect of RV16 infection on late responses}

Saline challenge did not significantly affect the recovery of histamine, tryptase, eosinophils, or TNF- $\alpha$ from late response BAL fluids of either allergic or normal subjects, nor was an effect of RV16 infection evident on these saline challenged segments (data not shown).

Histamine. Histamine was detectable in 8/15 late-response samples from normal, and 17/21 samples from allergic subjects. Bronchoalveolar lavage histamine concentrations $48 \mathrm{~h}$ after antigen challenge were augmented by RV16 infection $(P<0.001$, ANOVA), and were specifically greater in allergic subjects during the Acute Infection and post-infection study periods compared to the pre-infection period $(P<0.05$, Student NewmanKeuls test, both comparisons) (Fig. 2). In addition, significantly higher concentrations of histamine were detected in BAL fluids from allergic compared to normal individuals $(P<0.03$, ANOVA, Fig. 2), and a significant difference in the response to RV16 infection of allergic and normal subjects $(P=0.005)$ was observed: potentiation of histamine release $48 \mathrm{~h}$ after antigen challenge was seen in allergic, but not normal subjects.

Tryptase. Tryptase was undetectable in 4/15 samples from normal subjects, and was $\leq 0.4 \mathrm{ng} / \mathrm{ml}$ in all such samples. In samples from allergic subjects, tryptase was undetectable in 5/ 21 late response BAL fluids. Concentrations of tryptase in these BAL fluids were influenced neither by atopic status (normal $0.3 \pm 0.4$ vs. allergic $0.9 \pm 0.3 ; P>0.2$, ANOVA) nor by RV16 infection (pre-infection $0.5 \pm 0.4$, acute infection $1.0 \pm 0.4$, postinfection $1.1 \pm 0.4 ; P>0.65$, ANOVA).

Eosinophils. The late response of eosinophil recruitment to the airway after local antigen challenge is shown in Fig. 3. Increases in airway eosinophils $48 \mathrm{~h}$ after antigen challenge were not seen in normal subjects. In contrast, this finding was consistently observed in allergic individuals $(P<0.001$, ANOVA for effect of atopy). There was potentiation of eosinophil recruitment in relationship to RV16 infection $(P=0.005)$, and the enhancement was observed in allergic but not normal subjects $(P<0.03$, ANOVA, for difference in response to RV16 of allergics and normals). In addition, the study periods in which increased numbers of eosinophils were identified in allergic subjects compared to the pre-infection period were the acute infection and post-infection periods $(P<0.05$, Student Newman-Keuls test, both comparisons; Fig. 3).

$T N F-\alpha$. TNF- $\alpha$ concentrations were increased significantly in the late response of allergic compared to normal subjects $(P$ $<0.03$, ANOVA), and were further increased in association with RV16 infection ( $P=0.01$, ANOVA; Fig. 4). However, no significant dependence of the RV16 effect on atopy was observed $(P>0.3$, ANOVA for difference in response to RV16 of allergics and normals).

Summary of effects of RVI6 infection on late responses. Histamine release or persistent leakage into the airway and eosinophil recruitment were significantly increased by RV16 infection in allergic subjects, but not in normal volunteers 48 $h$ after segmental antigen challenge. TNF- $\alpha$ concentrations in BAL fluids obtained $48 \mathrm{~h}$ after antigen challenge were significantly increased in relationship to RV16 infection in both allergic and normal individuals.

\section{Discussion}

This study investigated the effects of RV16 infection on several indices of allergic airway inflammation, in both allergic and non-allergic subjects, using a reproducible human model of antigen induced airway inflammation (segmental bronchoprovocation with antigen) $(8,9)$. An experimental RV16 infection which did not alter airway physiology was associated with potentiated histamine release both immediately and $48 \mathrm{~h}$ after antigen challenge, and with increased recruitment of eosinophils to the airway $48 \mathrm{~h}$ after antigen challenge in allergic subjects. Moreover, these effects were apparent up to one month after RV16 inoculation in allergic subjects, and were associated with an increased concentration of TNF- $\alpha$ in BAL fluid $48 \mathrm{~h}$ after local antigen challenge.

Because of the close link between airway hyperresponsiveness and inflammation, $(24,25)$ previous observations of increased airway responsiveness during viral infection predict that 


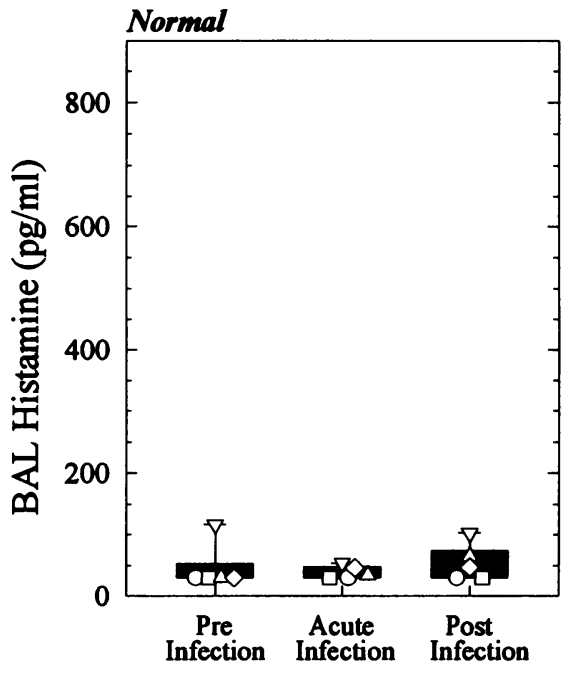

Study Period

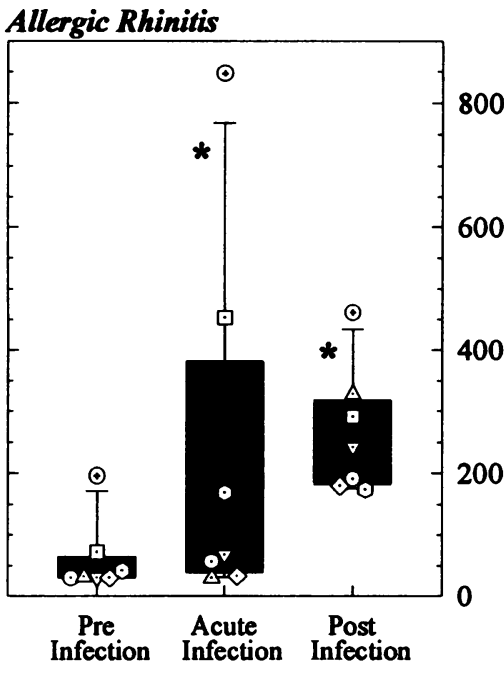

Study Period

Figure 2. BAL histamine concentrations $48 \mathrm{~h}$ after segmental antigen challenge in subjects experimentally infected with rhinovirus 16 . On the ordinate is the concentration of histamine observed in BAL fluids obtained $48 \mathrm{~h}$ after segmental antigen challenge (late response) in five normal volunteers (left panel, open symbols) and seven allergic rhinitis subjects (right panel, dotted symbols). Each individual is denoted by a unique symbol which is used consistently in Figs. 2-4 (e.g., the dotted diamond always represents the same individual). On the abscissa are the three study periods. The wide bars represent the 2575 percentiles, the whisker bars show the 5th and 95th percentiles, and the center crossbar shows the median. Asterisks and arrows denote statistically significant differences between post-infection and acute infection periods when compared with preinfection period by post hoc testing. There was a significant potentiating effect of RV16 infection, denoted as the study periods $(P<0.001$, ANOVA), with higher histamine concentrations observed during the Acute Infection and post-infection periods compared with pre-infection. There was also a significant difference in the BAL histamine concentration related to atopy ( $P<0.03$, ANOVA), with higher levels observed in allergic subjects. In addition, there was a significant difference in the response to RV16 infection depending on atopy $(P=0.005$, ANOVA), with allergic subjects exhibiting RV16 related potentiation of histamine release after segmental antigen challenge, whereas normal subjects did not.

inflammation should also be more intense. Lemanske et al. (6) reported both increased airway responsiveness to antigen, and an increased frequency of late phase allergic responses during experimental RV16 infection. Our current study provides direct evidence of enhanced antigen-induced mediator release and inflammation in the lower respiratory tract of allergic human subjects in response to RV16 infection, and may help to explain the development of late allergic reactions during a cold. However, due to the segmental nature of antigen deposition, direct assessments of physiologic airway obstruction and hyperrespon- siveness in close relationship to antigen challenge were not feasible in the present work. Nonetheless, these present data are entirely consistent with previous findings of increased plasma histamine after antigen challenge in allergic humans who developed new late phase responses to antigen challenge during RV16 infection, (7) and now provide direct evidence of persistent virally potentiated mediator release in the target organ (Fig. 2). Moreover, in the present study we were able to quantitate tryptase in BAL fluids from all allergic subjects after antigen challenge, whereas plasma tryptase was detectable in only one
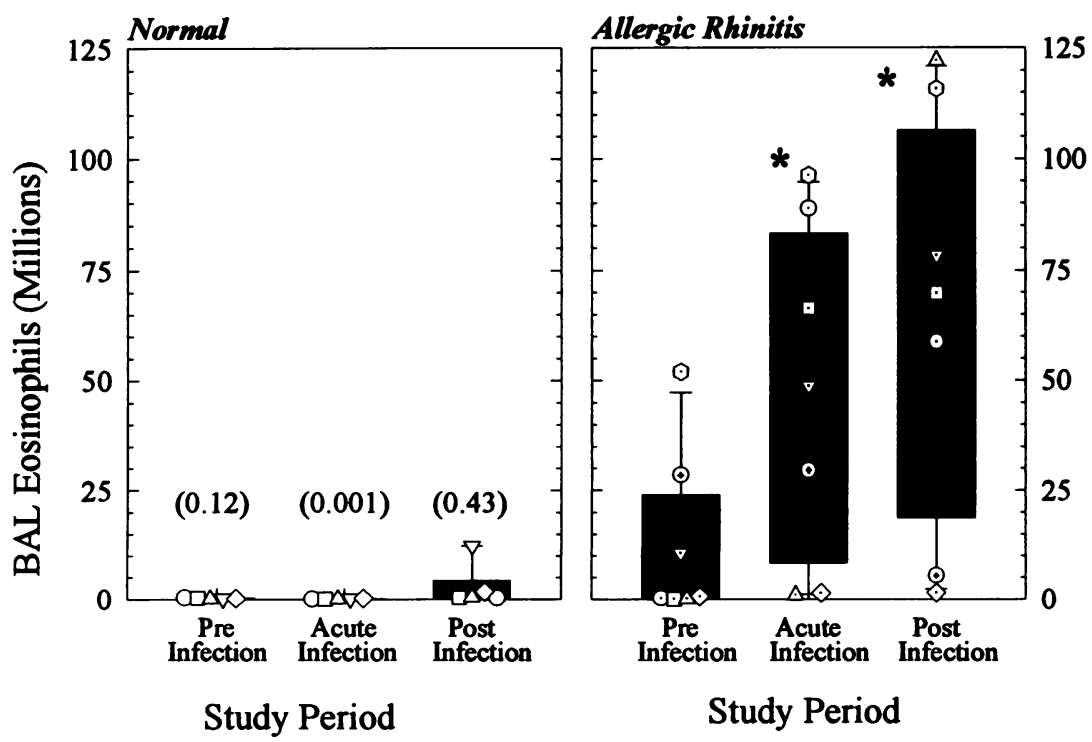

Figure 3. BAL eosinophils $48 \mathrm{~h}$ after segmental antigen challenge in subjects experimentally infected with rhinovirus 16 . On the ordinate is the number of eosinophils observed in BAL fluids obtained $48 \mathrm{~h}$ after segmental antigen challenge (late response) in five normal volunteers (left panel, open symbols) and seven allergic rhinitis subjects (right panel, dotted symbols). Each individual is denoted by a unique symbol which is used consistently in Figs. 2-4. On the abscissa are the three study periods. The wide bars represent the $25-75$ percentiles, the whisker bars show the 5th and 95th percentiles, and the center crossbar shows the median. Asterisks and arrows denote statistically significant differences between the post-infection and acute infection period when compared to pre-infection period by post hoc testing. Eosinophil recruitment in normal subjects was quantitatively small in all study periods. The actual median values are shown in parentheses. There was a significant potentiating effect of RV16 infection, denoted as the study periods $(P=$ 0.005 , ANOVA), and a significant difference in

overall eosinophil recruitment related to atopy $(P<0.001$, ANOVA), with allergic subjects exhibiting greater recruitment. In addition, there was a significant difference in the response to RV16 infection depending on atopy $(P<0.03$, ANOVA), with allergic subjects exhibiting heightened RV16 related antigen-driven eosinophil recruitment, whereas normal subjects did not. 


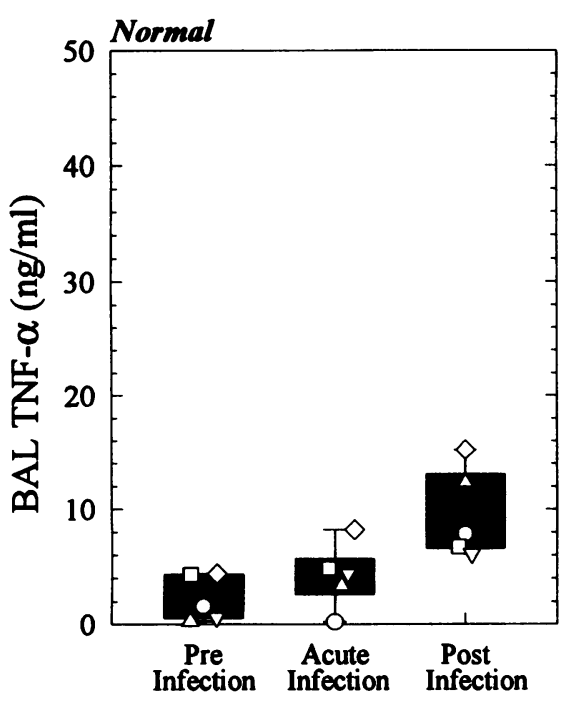

Study Period
Allergic Rhinitis

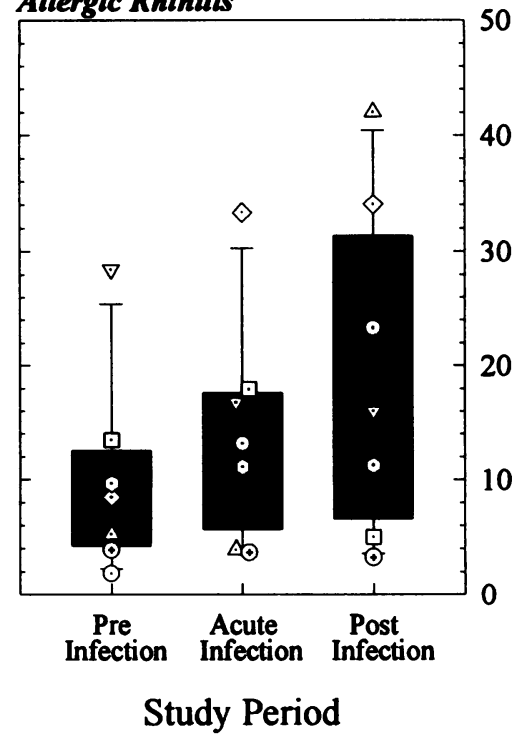

Figure 4. BAL TNF- $\alpha$ concentrations $48 \mathrm{~h}$ after segmental antigen challenge in subjects experimentally infected with rhinovirus 16 . On the ordinate is the concentration of TNF- $\alpha$ measured in BAL fluids obtained $48 \mathrm{~h}$ after segmental antigen challenge in five normal volunteers (open symbols) and seven allergic rhinitis subjects (dotted symbols). Each individual is denoted by a unique symbol which is used consistently in Figs. 2-4. On the abscissa are the three study periods. The wide bars represent the $25-$ 75 percentiles, the whisker bars show the 5th and 95th percentiles, and the center crossbar shows the median. A significant potentiating effect of RV16 infection, denoted as the study periods, was noted $(P=0.01$, ANOVA $)$ and can be seen as the upward trend of the median TNF- $\alpha$ response of allergic and normal individuals. There was also a significant difference in TNF- $\alpha$ concentration related to atopy, with higher concentrations observed in allergic subjects $(P<0.03$, ANOVA). However, in contrast to the significant interactive effects identified for histamine and eosinophils, there was no significant difference in the response to RV16 infection depending on atopy $(P>0.37$, ANOVA). subject in our previous work. Consequently, we can now more confidently ascribe mediator release in immediate fluids after antigen challenge to pulmonary mast cells, a suggestion consistent with previous reports $(9,11)$. However, the pronounced significant difference in histamine, but not tryptase, levels associated with viral infection could suggest that basophils contribute to the enhanced histamine release during and after viral infections.

Inflammation and late allergic reactions in asthma are characterized by prominent participation of eosinophils, which are observed in increased numbers in blood, sputum, bronchial tissue, and lung lavage fluids, (26) and which provoke pro-inflammatory responses from other cells (27-29). After segmental antigen challenge of allergic subjects, antigen-dose-dependent inflammation develops which is predominantly eosinophilic and macrophagic in nature $(8,9)$. It is particularly noteworthy, therefore, that antigen-induced eosinophil influx in late BAL fluids was so prominently increased both during and up to one month after RV16 infection (Fig. 3). These data suggest that viral effects on eosinophil recruitment, either direct or mediated by cytokines, are one mechanism by which late allergic reactions and increased airway hyperresponsiveness may be induced by viral upper respiratory tract infections.

It is important to note that RV16 did not directly cause or increase lower airway inflammation as assessed by BAL parameters, nor did it per se cause release of histamine. In both normal subjects and allergic rhinitis patients, there was no increase in histamine, eosinophils, or TNF- $\alpha$ concentration in the saline challenged segment (data not shown), nor were there RV16-associated changes in histamine or eosinophils in normal volunteers after antigen challenge. Hence, allergen-specific, rather than nonspecific inflammation was potentiated by RV16 inoculation. Collectively, the RV16 effect on airway inflammation appears to be principally an augmentation of allergic responses, a suggestion which is consistent with other current observations (5).

It was also noteworthy that with one exception, RV16 was not isolated from either BAL fluids or cells, despite the obvious potential for nasopharyngeal contamination of these specimens by bronchoscopy. In contrast, virus was always found in high titer in nasal washes (Table I). Thus, RV16 may not have been actively replicating in the lower respiratory tract of our subjects, despite clinical, immunologic, and virologic evidence of host infection. Nonetheless, significant potentiation of other measures of lower airway inflammation were observed. This observation suggests that mechanisms other than direct viral cytopathic effects in the lower respiratory tract may be important in augmenting airway inflammation from RV16 infection. In vitro experiments of viral infection of respiratory epithelium by Winther and colleagues (30) have led to similar conclusions. Thus, factors induced or produced by viral infection may mediate the potentiated inflammatory response.

In this regard, Naclerio et al. (31) suggested that kinins mediate nasal symptoms during experimental rhinovirus colds. In their study, increased levels of kinins, TAME-esterase, and albumin were observed in relationship to infection with RV39 or an untyped rhinovirus strain, $\mathrm{HH}$. Of interest, these investigators did not find increased nasal lavage histamine during the cold. However, Naclerio et al. did not challenge with antigen, a factor which probably explains the absence of histamine. Our data suggest that RV16 infection augments the allergic response, but has little direct effect on measures of airway inflammation, and are in that regard consistent. Moreover, in that Naclerio et al. used serial nasal lavage, and different RV serotypes, considerable differences exist in technique and experimental design between these two studies.

Cytokines are prime candidates to mediate the enhancement of antigen-driven airway inflammation. They prominently regulate the function of inflammatory cells, including eosinophils $(9,32)$ and macrophages, (33) and may also be induced by viral infections. In previous work, we demonstrated increased concentrations of TNF- $\alpha$ in BAL fluids of allergic subjects compared to healthy non-allergic volunteers $48 \mathrm{~h}$ after segmental antigen challenge (21). These and other factors may partici- 
pate in the functional up-regulation seen in airway cells after antigen challenge $(8,9)$. Our current data show that TNF- $\alpha$ is present in BAL fluids at concentrations high enough to modulate cell functions, particularly considering the 50-100-fold dilution of epithelial lining fluid cause by the BAL procedure. Rhinovirus infection in vivo has been associated with increased production of cytokines by human blood mononuclear cells in vitro, and with increased blastogenic responses to antigen (34). Our TNF- $\alpha$ data are consistent with these observations, and extend them to an in vivo human model, but do not establish the specific cellular source. Moreover, in that TNF- $\alpha$ concentrations were increased by RV16 infections in both normal and allergic subjects, other factors besides TNF- $\alpha$ must also participate in the development of airway inflammation. Thus, although our data on TNF- $\alpha$ should be viewed as a selective representation of the participation of the cytokine network rather than as a comprehensive picture, they nonetheless suggest that the cytokine network may be a key factor in regulating the enhanced airway allergic response.

It is conceivable that repetitive endobronchial antigen challenge, rather than RV16 infection, primed the allergic rhinitis subjects for an enhanced inflammatory response. We believe that this explanation is unlikely for several reasons. First, several of our infected subjects were studied again 3 mo after RV16 inoculation, and no further potentiation of inflammation was seen (data not shown). Second, additional experiments at 6 and 12 mo after inoculation in these same subjects have demonstrated return of the airway response to antigen to or towards the baseline, despite intervening segmental antigen challenges. Third, and most convincingly, repetitive endobronchial antigen challenge in four allergic individuals not inoculated with RV16 showed no enhancement of airway inflammatory responses (see Results). The statistical power of these last studies to identify a twofold increase in histamine was 0.93 , and to detect a fivefold increase in eosinophils was 0.63 .

Several other limitations of our study must be considered in applying these results to asthma. First, we chose to study subjects with allergic rhinitis for reasons outlined in prior publications $(6,7)$. Clear distinctions exist in the clinical presentation and course of asthmatics and rhinitis, which may reflect mechanistic differences in these groups of patients. Second, because of the invasive nature of the procedures, representative physiologic measurements could not be obtained contemporaneously with the BAL materials. Moreover, the concept of a late phase physiologic response to antigen is undefined using the technique of segmental antigen challenge. Nonetheless, our data provide new and direct evidence of the potentiating role of RV16 infection on antigen induced mediator release and airway inflammation.

In summary, RV16 infection results in persistently enhanced airway inflammation after local antigen challenge in allergic subjects, which persists for at least 1 mo after inoculation. Our observations suggest that viral infection is a critical determinant of this enhancement, but that mechanisms other than direct viral infection of the lower respiratory tract, including induction of pro-inflammatory cytokines, may be operative. Additional experiments will prove valuable to define more fully the mechanisms by which RV16 infection potentiates inflammation, bronchial obstruction, and wheezing in asthma.

\section{Acknowledgments}

The authors thank Carol A. Stevens, B.S., Kimberly A. Murphy, B.S., and Cheri A. Swenson, B.S. for technical assistance; Holly E. Reed,
B.S.N. R.N., Ann M. Dodge, B.S.N. R.N., and Gretchen M. Baehr, B.S.N. R.N., for nursing participation; Claire R. Dick, B.S.M.T. for inoculating subjects, obtaining nasal washes, and performing viral and serologic titrations; Joseph Costantino, Ph.D. for statistical advice and critique; and James H. Dauber, M.D., Nizar N. Jarjour, M.D., Robert F. Lemanske, Jr., M.D., and Bill T. Ameredes, Ph.D. for critical and thoughtful manuscript review.

This work was supported in part by National Institutes of Health (NIH)/National Heart, Lung, and Blood Institute grants K08-01828 and HL-44098, and American Lung Association of Wisconsin NIH/National Institute of Allergy and Infectious Diseases grant AI-026609.

\section{References}

1. Minor, T. E., E. C. Dick, A. N. DeMeo, J. J. Ouellette, M. Cohen, and C. E. Reed. 1974. Viruses as precipitants of asthmatic attacks in children. JAMA (J. Am. Med. Assoc.). 227:292-298.

2. Hall, W. J., C. B. Hall, and D. M. Speers. 1978. Respiratory syncytial virus infection in adults. Ann. Intern. Med. 88:203-205.

3. Busse W. W., and W. J. Calhoun. 1992. Infections. In Asthma: Basic Mechanisms and Clinical Management. P. J. Barnes, I. W. Rodger, and N. C. Thomson, editors. Academic Press, New York. 451-471.

4. Nicholson, K. G., J. Kent, and D. C. Ireland. 1993. Respiratory viruses and exacerbations of asthma in adults. Br. Med. J. 307:982-996.

5. Duff, A. L., E. S. Pomeranz, L. E. Gelber, G.W. Price, H. Farris, F. G. Hayden, T. A. E. Platts-Mills, and P. W. Heymann. Risk factors for acute wheezing in infants and children: viruses, passive smoke, and $\operatorname{IgE}$ antibodies to inhalant allergens. Pediatrics. 92:535-540.

6. Lemanske, R. F., E. C. Dick, C. A. Swenson, R. F. Vrtis, and W. W. Busse. 1989. Rhinovirus upper respiratory tract infection increases airway hyperreactivity and late asthmatic reactions. J. Clin. Invest. 83:1-10.

7. Calhoun, W. J., C. A. Swenson, E. C. Dick, L. B. Schwartz, R. F. Lemanske, Jr., and W. W. Busse. 1991. Experimental rhinovirus 16 infection potentiates histamine release after antigen bronchoprovocation in allergic subjects. Am. Rev. Respir. Dis. 144:1267-1273.

8. Calhoun, W. J., H. E. Reed, D. R. Moest, and C. A. Stevens. 1992. Enhanced superoxide production by alveolar macrophages and airspace cells, airway inflammation, and alveolar macrophage density changes after segmental antigen bronchoprovocation in allergic subjects. Am. Rev. Respir. Dis. 145:315-325.

9. Sedgwick, J. B., W. J. Calhoun, G. J. Gleich, H. Kita, J. S. Abrams, L. B. Schwartz, B. Volovitz, M. Ben-Yaakov, and W. W. Busse. 1991. Immediate and late airway responses of allergic rhinitis patients to segmental antigen challenge. Am. Rev. Respir. Dis. 144:1274-1281.

10. Liu, M. C., W. C. Hubbard, D. Proud, B. A. Stealey, S. J. Gallli, A. Kagey-Sabotka, E. R. Bleeker, L. M. Lichtenstein. 1991. Immediate and late inflammatory responses to ragweed antigen challenge of the peripheral airway in allergic asthmatics. Am. Rev. Respir. Dis. 144: 51-58.

11. Wenzel, S. E., A. A. Fowler, III, and L. B. Schwartz. 1988. Activation of pulmonary mast cells by bronchoalveolar allergen challenge. In vivo release of histamine and tryptase in atopic subjects with and without asthma. Am. Rev. Respir. Dis. 137:1002-1008.

12. Ohnishi, T., H. Kita, D. Weiler, J. B. Sedgwick, W. J. Calhoun, W. W. Busse, J. S. Abrams, and G. J. Gleich. 1993. IL-5 is the predominant eosinophilactive cytokine in the antigen-induced pulmonary late phase reaction. Am. Rev. Respir. Dis. 147:901-907.

13. Settipane, G. A. 1991. Rhinitis: introduction. In Rhinitis. 2nd edition. G. A. Settipane, editor. OceanSide Publications, Providence, RI. 1-11.

14. Meschievitz, C. K., S. B. Schultz, and E. C. Dick. 1984. A model for obtaining predictable natural transmission of rhinoviruses in human volunteers. J. Infect. Dis. 150:195-201.

15. Calhoun, W. J., and R. K. Bush. 1990. Enhanced reactive oxygen species metabolism of airspace cells and airway inflammation follow antigen challenge in human asthma. J. Allergy Clin. Immunol. 86:306-313.

16. Chai, H., R. Farr, and L. A. Froehlich. 1975. Standardization of bronchial inhalation challenge procedures. J. Allergy Clin. Immunol. 56:323-327.

17. The BAL Cooperative Study Group. 1990. Bronchoalveolar lavage constituents in healthy individuals, idiopathic pulmonary fibrosis, and selected comparison groups. Am. Rev. Respir. Dis. 141:S169-S202.

18. Bowsher, R. R., K. M. Verburg, and D. P. Henry. 1983. Rat histamine Nmethyltransferase: quantitation, tissue distribution, purification, and immunologic properties. J. Biol. Chem. 258:12215-12220.

19. Schwartz, L. B., T. R. Bradford, D. G. Lee, and J. F. Chlebowski. 1990 Immunologic and physicochemical evidence for conformational changes occurring on conversion of human mast cell tryptase from active tetramer to inactive monomer: production of monoclonal antibodies recognizing active tryptase. $J$. Immunol. 144:2304-2311. 
20. Macy, E., M. Kemeny, and A. Saxon. 1988. Enhanced ELISA: how to measure less than 10 picograms of a specific protein (immunoglobulin) in less than 8 h. FASEB (Fed. Am. Soc. Exp. Biol.) J. 2:3003-3009.

21. Calhoun, W. J., K. Murphy, C. A. Stevens, N. N. Jarjour, and W. W Busse. 1992. Increased interferon- $\gamma$ and tumor necrosis factor- $\alpha$ in bronchoalveolar lavage fluid after antigen challenge in allergic subjects. Am. Rev. Respir. Dis. 145:638a. (Abstr.)

22. Glantz, S. A. 1992. Primer of Biostatistics. 3rd edition. McGraw-Hill, Inc. New York.

23. Armitage, P., and G. Berry. 1989. Statistical Methods in Medical Research 2nd edition. Blackwell Scientific Publications, Boston.

24. Nadel, J. A. 1983. Bronchial reactivity. Adv. Intern. Med. 28:207-225.

25. Reed, C. E. 1986. New therapeutic approaches in asthma. J. Allergy Clin. Immunol. 77:537-543.

26. Bousquet, J., P. Chanez, J. Y. Lacoste, G. Barneon, N. Ghavanian, I Enander, P. Venge, S. Ahlstedt, J. Simony-Lafontane, P. Godard, and F.-B. Michel. 1990. Eosinophilic inflammation in asthma. N. Engl. J. Med. 323:10331039.

27. Rankin, J. A., P. Harris, and S. J. Ackerman. 1992. The effects of eosinophil-granule major basic protein on lung-macrophage superoxide anion generation J. Allergy Clin. Immunol. 89:746-752.

28. O'Donnell, M. C., S. J. Ackerman, G. J. Gleich, and L. L. Thomas. 1983.
Activation of basophil and mast cell histamine release by eosinophil major basic protein. J. Exp. Med. 157:1981-1991.

29. Moy, J. N., G. J. Gleich, and L. L. Thomas. 1990. Noncytotoxic activation of neutrophils by eosinophil granule major basic protein: effect on superoxide generation and lysosomal enzyme release. J Immunol. 145:2626-2632.

30. Winther, B., J. M. Gwaltney, and J. O. Handley. 1990. Respiratory virus infection of monolayer cultures of human nasal epithelial cells. Am. Rev. Respir. Dis. 141:839-845.

31. Naclerio, R. M., D. Proud, L. M. Lichtenstein, A. Kagey-Sobotka, J. O. Hendley, J. Sorrentino, and J. M. Gwaltney. 1988. Kinins are generated during experimental rhinovirus colds. J. Infect. Dis. 157:133-142.

32. Silberstein, D. S., K. F. Austen, and W. F. Owen. 1989. Hemopoietins for eosinophils. Glycoprotein hormones that regulate the development of inflammation in eosinophilia-associated disease. Hematol. Oncol. Clin. N. Am. 3:511533.

33. Schreiber, R. D., and A. Celada. 1985. Molecular characterization of interferon- $\gamma$ as a macrophage activating factor. In Lymphokines 11. E. Pick, editor. Harcourt Brace Jovanovich, New York. 87-118.

34. Hsia, J, A. L. Goldstein, G. L. Simon, M. Sztein, and F. G. Hayden. 1990 Peripheral blood mononuclear cell interleukin- 2 and interferon- $\gamma$ production, cytotoxicity, and antigen-stimulated blastogenesis during experimental rhinovirus infection. J. Infect. Dis. 162:591-597. 